\title{
Visualization of surface electronic structure: Dispersion of surface states of $\mathrm{Ag}(110)$
}

\author{
J. I. Pascual, ${ }^{1, *}$ Z. Song ${ }^{1}$ J. J. Jackiw, ${ }^{1,2}$ K. Horn, ${ }^{1}$ and H.-P. Rust ${ }^{1}$ \\ ${ }^{1}$ Fritz-Haber-Institut der Max-Planck-Gesellschaft, Faradayweg 4-6, D-14195 Berlin, Germany \\ ${ }^{2}$ Department of Chemistry, The Pennsylvania State University, University Park, Pennsylvania 16802-6300
}

(Received 22 December 2000; revised manuscript received 16 March 2001; published 1 June 2001)

\begin{abstract}
Scanning tunneling spectroscopy is used to examine unoccupied surface states of $\operatorname{Ag}(110)$. We demonstrate how Fourier transformation of conductance data can be used to determine dispersion throughout the entire surface Brillouin zone, at energies up to the vacuum level. Considerable improvement of previous inverse photoemission data is obtained. Additional features in the Fourier transformed images are found accompanying those states lying in the proximity the Brillouin zone boundary. Their origin is related to the coherent interference of surface state-derived Bloch waves induced by the non-negligible crystal potential, as we determine on the basis of model calculations.
\end{abstract}

DOI: 10.1103/PhysRevB.63.241103

PACS number(s): 73.20.At, 68.37.Ef, 71.18.+y

The unoccupied surface states of the (110) noble metal surfaces are crucial for an understanding of their interesting optical and electronic properties; for example, single particle excitations into these states lead to an anisotropy in their surface electric susceptibility, and consequently, in their optical reflectance. ${ }^{1}$ In these surfaces, the projection of the inverted bulk band gap located at the bulk $L$ point is centered at the $\bar{Y}$ point, on the boundary of the (110) surface Brillouin zone (SBZ). There, two surface states exist, also centered at the $\bar{Y}$ point. Traditionally, photoelectron spectroscopy and inverse photoemission (IP) are used to determine energies and dispersion relations $E\left(\mathbf{k}_{\|}\right)$of surface states. ${ }^{2}$ Here, we examine the unoccupied electronic structure of $\operatorname{Ag}(110)$ using scanning tunneling spectroscopy (STS).

Scanning tunneling microscopy (STM) and spectroscopy have proven to be valid tools to access information about surface-state dynamics. For isotropic surface states, STS has demonstrated high energy resolution through the determination of the states parabolic dispersion relation, $E\left(\mathbf{k}_{\|}\right),{ }^{3-6}$ and deviations arising when the state wave vector approaches the symmetry points at the SBZ boundary. ${ }^{7}$ For the case of anisotropic surfaces (like the one used here), STM has also shown its capability to resolve information in $\mathbf{k}$ space at the Fermi energy by drawing the shape of a surface state's Fermi contour $^{8}$ and higher-order Bloch components. ${ }^{9,10}$ In our study of the $\operatorname{Ag}(110)$ surface we combine both the energy resolution shown for the isotropic states and the wave-vector resolution demonstrated for the anisotropic states and obtain a detailed description of the dispersion of its $S_{2}$ unoccupied surface state (Fig. 1). The analysis is extended to wave vectors over the entire Brillouin zone and to energies up to the vacuum level $\left(E_{v}\right)$, above which the electronic transport is governed by field emission.

The existence of the unoccupied $\mathrm{S}_{2}$ surface state was predicted by Ho et al. ${ }^{11}$ with self-consistent pseudopotential calculations to explain the optical anisotropy of this surface. ${ }^{1}$ From inverse photoemission studies, ${ }^{12-14}$ it was determined that the minimum energy of the $S_{2}$ state is between 1.6 and $1.7 \mathrm{eV}$ above the Fermi level $E_{F}$, and disperses along the $\overline{\Gamma Y}$ direction with an effective mass between $0.7 m_{e}$ (Ref. 11) and $0.9 m_{e}$ (Ref. 14). The shape of the surface state in the
(110) projected bulk band as determined from these previous studies is presented in Fig. 1(a), together with a surface-state contour in reciprocal space. From our results, we find that a simple free-electron-like picture cannot be used to describe the dispersing behavior of this surface state. A strong deviation from a parabolic behavior is observed close to the zone boundary. There, additional features appear in our data, which are interpreted, with the help of a model calculation, as arising from the non-negligible effect of the lattice on the quasiparticle dynamics in this region of reciprocal space.

Experiments were performed in an ultrahigh vacuum scanning tunneling microscope in thermal equilibrium with a liquid helium bath. ${ }^{16}$ Besides a high $(\approx 2 \mathrm{meV})$ energy resolution, at such low temperature $(\sim 4 \mathrm{~K})$, the instrument possesses sufficient stability to allow long recording times for the measurement of the tunneling junction conductance $(d I / d V)$ using lock-in amplification techniques. The silver single-crystal was cleaned following standard procedures: several cycles of $\mathrm{Ar}^{+}$sputtering $(1 \mathrm{keV})$ and annealing at $\sim 800 \mathrm{~K}$ produced a clean surface with an impurity density smaller than $10^{-2} \mathrm{~nm}^{-2}$.

In the proximity of surface defects, coherent interference of surface states gives rise to oscillations in the local density of states (LDOS), which can be directly correlated with oscillations in the junction conductance $(d I / d V)$. These realspace oscillations carry unambiguous information about the
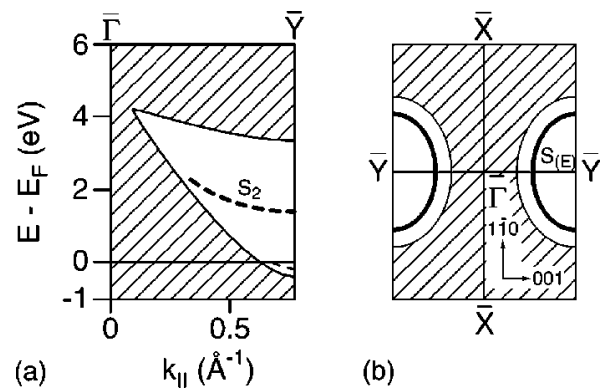

(b)

FIG. 1. (a) $S_{2}$ surface-state location in the (110) projected bulk band diagram along the $\overline{\Gamma Y}$ reciprocal space direction, reconstructed from data in Refs. 11-14. (b) Schematic of the elliptical surface-state contour at the energy $2.4 \mathrm{eV}$ above $E_{F}$ as obtained from our STS measurement Ref. 15. 


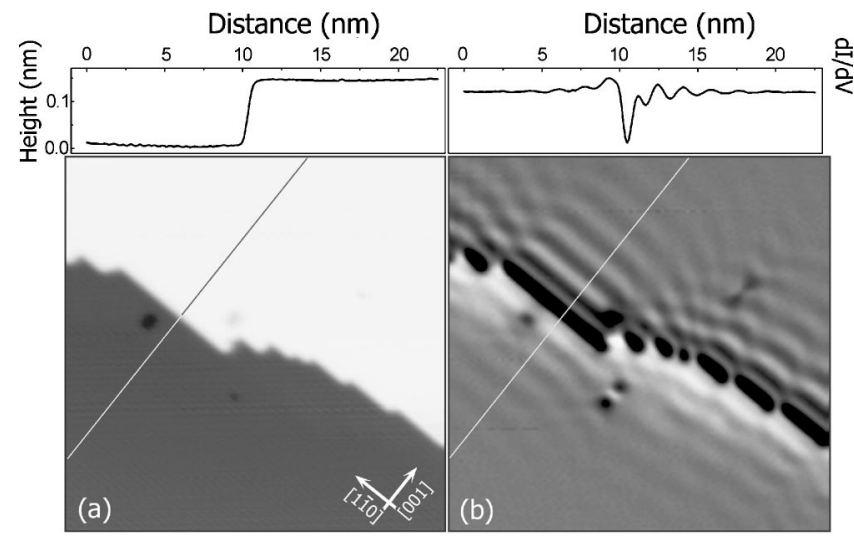

FIG. 2. (a) Constant current STM image. (b) $d I / d V$ spatial distribution around a monoatomic step in the $\operatorname{Ag}(110)$ surface. The plots above each image represent profiles along the lines indicated in the figures. $\left(20 \mathrm{~nm} \times 20 \mathrm{~nm} ; V_{s}=4 \mathrm{~V} ; I=8 \mathrm{nA} ; V_{a c}=25 \mathrm{mV}\right.$ rms @ $2.1 \mathrm{KHz}$; measurement time is $20 \mathrm{~min}$.)

dynamics of the surface states. At very low bias, it was demonstrated that such interference patterns can be directly observed in STM images and that their wavelengths are half the Fermi wavelength. ${ }^{17,18}$ At the high sample bias needed to access the $\mathrm{S}_{2}$ surface state $\left(V_{s} \geqslant 1.7 \mathrm{eV}\right)$, the topography of a constant current STM image integrates multiple wavevector contributions in the energy window from $E_{F}$ to $E_{F}$ $+\mathrm{e} V_{s}$. Therefore, in order to resolve the LDOS oscillations at the energy $\mathrm{e} V_{s}$ (above $E_{F}$ ), the tunnel junction conductance $(d I / d V)$ must be measured directly. ${ }^{19}$ This measurement is done by adding a small modulation signal $V_{a c} \cos (\omega t)$ to the selected sample bias $V_{s}$. The amplitude of the first harmonic in the resulting modulation of the tunneling current is proportional to the magnitude of $d I /\left.d V\right|_{V_{s}}$. In all the results presented here, the conductance $d I / d V$ was measured with the feedback loop closed. To prevent the modulation in the tunneling current from affecting the tunnel junction width, the frequency of $V_{a c}$ (generally over $2 \mathrm{KHz}$ ) was set above the cutoff frequency of the low-pass filter of the feedback loop electronics.

A comparison of a STM image of the region around a monatomic step with the spatial distribution of $d I /\left.d V\right|_{V_{s}}$ in that same area is shown in Fig. 2. At the top and the bottom of the step edge the conductance image [Fig. 2(b)] shows a pattern of waves preferentially along the [100] direction, which is not seen in the STM image in Fig. 2(a). Therefore, we can rule out the possibility that oscillations of the tunnel barrier width (topography) distort the direct assignment of the measured conductance with the LDOS. ${ }^{5,20}$

Figure 2(b) also shows that the interference pattern is rather anisotropic; ${ }^{15}$ there is a preferred orientation of the standing waves which coincides with the $\overline{\Gamma Y}$ direction in reciprocal space. On the basis of the Brillouin-zone topology in Fig. 1, the oscillations in the conductance shown in Fig. 2(b) are identified as interference of the $S_{2}$ surface states in the proximity of the step edge. This assignment is clearly in conflict with the previous IP results, since the image in Fig. 2(b) was measured at $e V_{s}=4.0 \mathrm{eV}$, well above the energy where the $\mathrm{S}_{2}$ states cross a bulk band, as it was found in these IP experiments $(2.5 \mathrm{eV})$. It is, therefore, desirable to extend the analysis of this surface state to a large range of energies. In this way, the evolution of the conductance oscillation shape and characteristic wavelengths with energy will describe the dispersion relation of the $\mathrm{S}_{2}$ state.

To analyze the dispersion in the $\overline{\Gamma Y}$ direction, we have chosen a straight step edge along the [1 $1 \overline{1} 0]$ direction, which acts as a wall for one-dimensional scattering of the surface states. Measurements were performed on the upper terrace near the step due to the higher reflection coefficients for electrons impinging from this terrace. ${ }^{21}$ The tip was set to raster in the direction perpendicular to the step edge with the feedback loop closed while $d I /\left.d V\right|_{V_{s}}$ was recorded from the lock-in amplifier. For one energy value $e V_{s}$, a conductance profile was obtained from an average of 50 individual scans across the step edge. We have repeated this procedure for $V_{s}$ ranging from $1.5 \mathrm{~V}$ to $4.3 \mathrm{~V}$ in steps of $25 \mathrm{mV}$. The results are shown in Fig. 3(a), where the junction conductance is represented by a gray scale, plotted as a function of the distance from the step edge (horizontal axis) and the energy (vertical axis). The pattern constructed thus gives evidence of a continuous increase of the characteristic wavelength of

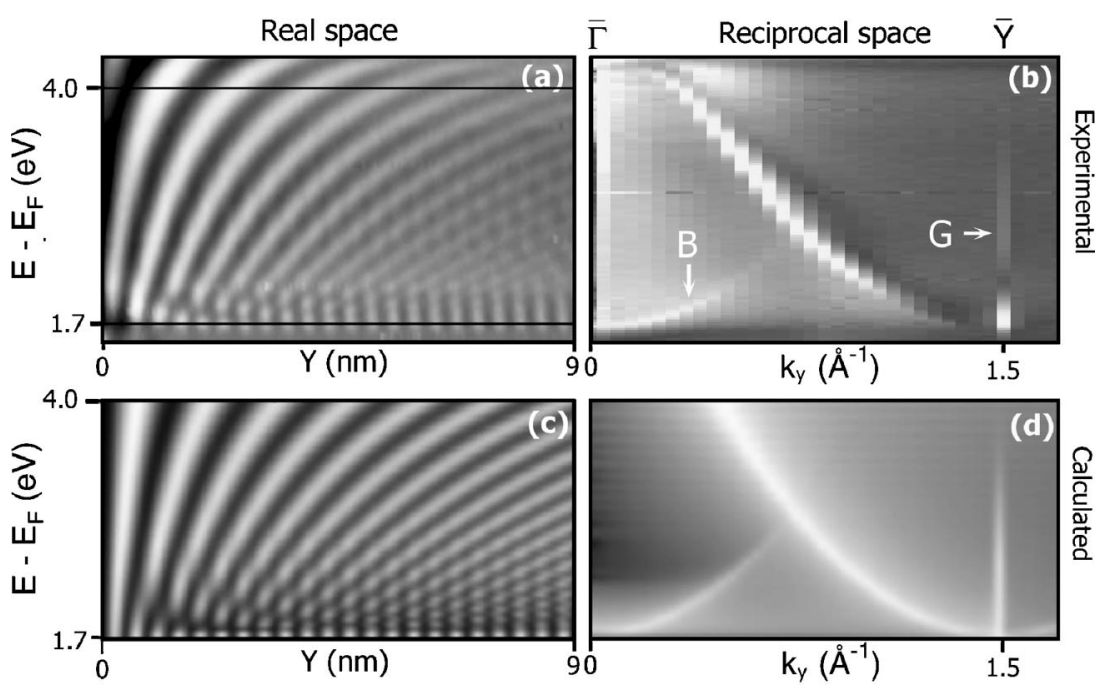

FIG. 3. (a) Gray scale representation of experimental $d I / d V$ data as a function of the energy $\left(E-E_{F}=e V_{s}\right)$ and the distance $(Y)$ from a monoatomic step parallel to the (1 $\overline{1} 0)$ direction. (b) one-dimensional FT of $d I /\left.d V\right|_{V_{s}}$ vs $Y$ data represented in a gray scale. The horizontal axis, representing $\mathbf{k}$ space, is (twice) the $\overline{\Gamma Y}$ axis of the reciprocal space (indicated above). ( $I=7 \mathrm{nA}$;

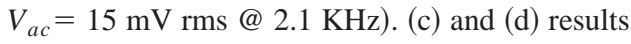
from the calculations described in the text, represented similarly to Figures 3(a) and 3(b). In both experiment and calculations the tip-sample distance increases linearly with energy $3.5 \AA$. An initial position of $4 \AA$ is assumed in the calculations, together with a ratio $U_{-1}=U_{0}=U_{1}$ and an effective mass $m^{*}=0.5 m_{e}$. 
the conductance waves (decrease of the surface state wave vector) with energy. This behavior defines the surface state's dispersion relation $E\left(\mathbf{k}_{\mathbf{y}}\right)$ characteristic of its position in reciprocal space.

In the data of Fig. 3(a), scattering occurs only for the wave-vector components along the $\overline{\Gamma Y}$ direction, reducing the problem to one dimension. In order to evaluate the dispersion relation of the $S_{2}$ surface state, we have calculated the one-dimensional Fourier transformation (FT) of the spatial one-dimensional conductance profiles of Fig. 3(a). In analogy with the FT of $2 \mathrm{D}$ conductance images, ${ }^{18,22,23}$ this mathematical procedure can resolve, for a particular energy, the different periodic components involved in real-space conductance oscillations, and hence in the LDOS. This periodic components can thus be directly related with twice the surface-state wave vectors. Based upon this argument, in Fig. 3(b) we show the results from the 1D FT of the conductance profiles in a gray scale. There, the vertical axis remains the same, while the horizontal axis (above) is adjusted to represent the $\overline{\Gamma Y}$ direction in reciprocal space.

The main feature in the experimental 1D FT data [Fig. 3(b)] is a branch dispersing from $\bar{Y}$ at approximately $1.7 \mathrm{eV}$, and reaching the zone center at $4.1 \mathrm{eV}$. This feature can be directly related to the $S_{2}$ state. However, two additional features occur in reciprocal space: a dispersing branch ' $B$ ", centered at the $\bar{\Gamma}$ point accounts for the interferencelike pattern observed at low energy in the real space diagram of Fig. 3(a). A nondispersing feature " $G$ ', occurs at the reciprocal lattice point $\bar{Y}\left[k_{\bar{Y}}=\pi / a\right.$, where $a=4.09 \AA$ is the lattice constant along the (100) direction]. As we will show in the following discussion, these features arise from the influence of the underlying atomic periodic potential on the dynamics of the quasiparticle.

A simple model is used to explain the origin of features $G$ and $B$ in the reciprocal space representation and to demonstrate the validity of the 1D FT method to resolve the correct dispersion behavior of surface state with such anisotropic configuration in reciprocal space. We simulate the junction conductance by evaluating the LDOS at the tip position for states with the following wave function: ${ }^{19}$

$$
\psi\left(\mathbf{r}_{\|}, z\right)=\sum_{G} U_{G} e^{i\left(\mathbf{k}_{\|}-\mathbf{G}\right) \mathbf{r}_{\|}} e^{-\kappa_{z}\left(\mathbf{k}_{\|}-\mathbf{G}, E\right) z}
$$

Here, $\kappa_{z}\left(\mathbf{k}_{\|}-\mathbf{G}, E\right)$ is the vacuum decay length for the wave vector $\mathbf{k}_{\|}-\mathbf{G}$ at energy $E$, and $\mathbf{G}$ is a reciprocal lattice vector. In the calculations, the summation in Eq. (1) is limited to first-order terms $(G=-1,0,1)$ (Ref. 9) along $\overline{\Gamma Y}$. In this way, the wave function is the solution of a free-electron Hamiltonian plus a sinusoidal potential term with the lattice periodicity [along the (100) direction], the simplest case that introduces the effect of a periodic potential in the dynamics of free electrons. Approximating the step by an infinite potential barrier for the sake of simplicity, the LDOS in the proximity of steps is then readily calculated as the coherent sum of the Bloch plane waves from Eq. (1), traveling in both directions:
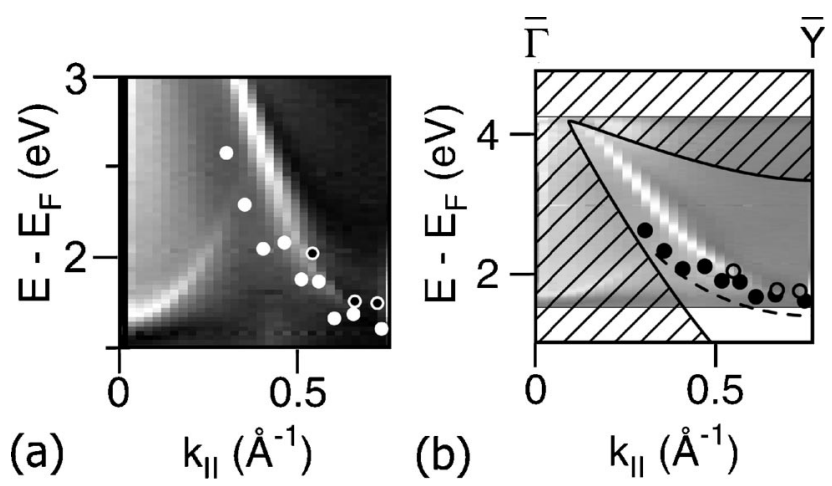

FIG. 4. Comparison of the experimental data of Fig. 3 with previous IP results and calculations (dashed line from Ref. 11, empty circles from Ref. 12, and filled from Ref. 14). In (a) the whole energy range of the STS measurements is inserted in the projected bulk band diagram of (a). (b) Zoom of the low-energy region.

$$
\rho(E, y)=\oint_{S(E)}\left|\psi\left(r_{\|}, z, k_{y}>0\right)+\psi\left(r_{\|}, z, k_{y}<0\right)\right|^{2} .
$$

This integral is evaluated numerically at the energy $E$ $=e V_{s}$, along the surface state contour $S(E)$ (Fig. 1), which has dimensions along the $\overline{\Gamma Y}$ direction defined by the relation $E\left(\mathbf{k}_{\mathbf{y}}\right)$. The result of this calculation and its 1D FT representation are shown in Fig. 3(c) and 3(d), for an effective mass of $0.5 m_{e}$. This simulation succeeds in reproducing the general shape of the main parabolic feature observed in the experimental data. Furthermore, the shape of this parabola coincides exactly with the relation $E\left(\mathbf{k}_{\mathbf{y}}\right)$ introduced in the calculations, showing that the 1D FT method is valid to directly and quantitatively derive the relation $E\left(\mathbf{k}_{\|}\right)$.

The model also reproduces features $G$ and $B$ observed in the experimental data. Their origin is exclusively related to the dynamics of a quasiparticle in a periodic lattice; none of these features are observed when only the 0th order term in Eq. (1) (free-electron case) is used in the simulation. We find that the experiments are reproduced when the first-order terms are included in calculation and it is by the coherent interference of the resulting three Bloch waves that the additional features appear in the results. In particular, interference of the $G=0$ and $G=-1$ plane waves give rise to a term with the periodicity of the atomic lattice along the (100) direction $\left[\cos \left(2 k_{\bar{Y}} y\right)\right]$ and with an amplitude modulated by $\cos \left[2\left(k_{y}-k_{\bar{Y}}\right) y\right],{ }^{10}$ which are responsible for the features $G$ and $B$, respectively. Therefore, in reciprocal space representation, feature $B$ represents the surface-state parabola shifted by $k_{\bar{Y}}$ (Refs. 9 and 10) and its appearance is mainly dictated by the $U_{-1}$ coefficient, which reflects the importance of the underlying periodic potential. In the calculation of Fig. 3, the $U_{-1} / U_{0}$ ratio used is 1 .

Since the 1D FT method is demonstrated to be a valid method for extracting surface-state dispersion relations, in Fig. 4 we can directly compare the results from our STS measurements with the inverse photoemission results. For energy values below $2.5 \mathrm{eV}$ [Fig. 4(b)], both coincide in detail. From our measurements we obtain a quasiparticle ef- 
fective mass of $0.7 m_{e}$, which is in reasonable agreement with the calculations of Ho et al. ${ }^{11}$ However, above this energy [Fig. 4(a)], the surface state clearly deviates from this behavior, and instead of crossing the bulk band edge, the surface state rises close to it up to the vacuum level.

The effect of a periodic lattice on the quasiparticle dynamics gives rise to deviations from a parabolic dispersion. A weak periodic potential has its major effect on those states with wave vectors close to the ones at which Bragg reflections can occur. ${ }^{24}$ In the results presented above, we observe clear indications of such deviations from a free-electron picture at the bottom of the $S_{2}$ band, where the states' wave vectors are in the proximity of the $\bar{Y}$ point. In that energy region, the surface band flattens as it approaches the reciprocal space symmetry point $(\bar{Y})$, defining an increment in the quasiparticle effective mass from $0.5 m_{e}$ to $0.7 m_{e}$. There, the lattice effect also manifests itself in our results with the appearance of a shifted replica of the surface-state feature, which is an evidence of the nonvanishing effect of the underlying periodic potential in that region of the $\mathbf{k}$ space.

In the top part of the $\mathrm{S}_{2}$ band, as the band approaches the $\Gamma$ point, we observe a similar flattening of the reciprocal space feature [Figs. 3(b) and 4(a)]. The smallest wave vector clearly measured is $0.12 \AA^{-1}$. Beyond this point, the surface-state probably crosses the gap edge before reaching the $\bar{\Gamma}$ point, after having dispersed over $85 \%$ of the SBZ.

In summary, we have examined the topology of the $\mathrm{S}_{2}$ unoccupied surface state of $\mathrm{Ag}(110)$ in reciprocal space using scanning tunneling spectroscopy, showing its unique capabilities to resolve surface state dynamics at energies up to the vacuum level and for wave vectors in the entire Brillouin zone. In this study we find that the underlying atomic lattice, far from being "seen" in the data, induces a decrease of the dispersing behavior of the states as they approach the high symmetry points of the Brillouin zone and gives rise to additional features in reciprocal space arising from the coherent interference of the surface state derived Bloch waves. A Fourier transformation of conductance data can be used to successfully reconstruct the dispersion of surface states in reciprocal space.

We acknowledge discussions with H. Conrad and P.S. Weiss. J.I.P. is indebted to the European Union for a research grant under the "Marie Curie" scheme. Z.S. and J.J.J. also thank the Max-Planck-Gesellschaft and the U.S. National Science Foundation (J.J.J.) for support of visits to the FritzHaber-Institut.
*Corresponding author. Email address: pascual@fhi-berlin.mpg.de

${ }^{1}$ T. E. Furtak and D. W. Lynch, Phys. Rev. Lett. 35, 960 (1977).

${ }^{2}$ Angle-Resolved Photoemission, edited by S.D. Kevan, Studies in Surface Science and Catalysis Vol. 74 (Elsevier, Amsterdam, 1992).

${ }^{3}$ M. F. Crommie, C. P. Lutz, and D. M. Eigler, Nature (London) 363, 524 (1993).

${ }^{4}$ Y. Hasegawa and Ph. Avouris, Phys. Rev. Lett. 71, 1071 (1993).

${ }^{5}$ J. Li, W.-D. Schneider, and R. Berndt, Phys. Rev. B 56, 7656 (1997).

${ }^{6}$ O. Jeandupeux, L. Bürgi, A. Hirstein, H. Brune, and K. Kern, Phys. Rev. B 59, 15926 (1999).

${ }^{7}$ L. Bürgi, L. Petersen, H. Brune, and K. Kern, Surf. Sci. 447, L157 (2000).

${ }^{8}$ Ph. Hofmann, B. G. Briner, M. Doering, H.-P. Rust, E. W. Plummer, and A. M. Bradshaw, Phys. Rev. Lett. 79, 265 (1997).

${ }^{9}$ B. G. Briner, Ph. Hofmann, M. Doering, H.-P. Rust, E. W. Plummer, and A. M. Bradshaw, Europhys. Lett. 39, 67 (1997); Phys. Rev. B 58, 13931 (1998).

${ }^{10}$ L. Peterson, B. Shaefer, E. Laesgaard, I. Stensgaard, and F. Besenbacher, Surf. Sci. 457, 319 (2000).

${ }^{11}$ K.-M. Ho, B. N. Harmon, and S. H. Liu, Phys. Rev. Lett. 44, 1531 (1980).

${ }^{12}$ B. Reihl, R. R. Schlittler, and H. Neff, Phys. Rev. Lett. 52, 1826
(1984).

${ }^{13}$ A. Goldmann, V. Dose, and G. Borstel, Phys. Rev. B 32, 1971 (1985).

${ }^{14}$ W. Altmann, V. Dose, and A. Goldmann, Z Phys. B: Condensed Matter 65, 171 (1986).

${ }^{15}$ A two-dimensional Fourier transformation of conductance image like the one in Fig. 2(b) reproduces part of the elliptical surface state contour, which is centered in the $\bar{Y}$ point; (unpublished).

${ }^{16}$ H.-P. Rust, J. Buisset, E. K. Schweizer, and L. Cramer, Rev. Sci. Instrum. 68, 129 (1997).

${ }^{17}$ P. T. Sprunger, L. Peterson, E. W. Plummer, E. Laesgaard, and F. Besenbacher, Science 275, 1764 (1997).

${ }^{18}$ L. Peterson et al., Phys. Rev. B 57, R5868 (1998).

${ }^{19}$ J. Tersoff and D. R. Hamann, Phys. Rev. Lett. 50, 1998 (1983); Phys. Rev. B 31, 805 (1985).

${ }^{20}$ G. Hörmandinger, Phys. Rev. B 49, 13897 (1994).

${ }^{21}$ L. Bürgi, O. Jeandupeux, A. Hirstein, H. Brune, and K. Kern, Phys. Rev. Lett. 81, 5370 (1998).

${ }^{22}$ Z. Song, J. I. Pascual, H. Conrad, K. Horn, and H.-P. Rust, Appl. Phys. A (to be published).

${ }^{23}$ J. Kliewer, Ph.D. thesis, RWTH Aachen, 2000.

${ }^{24}$ N. W. Ashcroft and N. D. Mermin, Solid State Physics (Saunders College, Philadelphia, 1976). 